\title{
Entrenamiento en remediación cognitiva y habilidades emocionales en formato grupal para pacientes con obesidad: Un estudio piloto
}

\author{
Mara Segura $^{1}$, María Roncero ${ }^{1}$, Javier Oltra-Cucarella ${ }^{1}$, Lorena Blasco ${ }^{2}$, Sonia Ciscar ${ }^{2}$, \\ Mónica Portillo ${ }^{2}$, Amparo Malea ${ }^{3}$, Raúl Espert ${ }^{1}$ y Conxa Perpiñá ${ }^{1,4}$ \\ ${ }^{1}$ Universidad de Valencia, Valencia, España. \\ ${ }^{2}$ Hospital de la Ribera, Alzira Valencia, España. \\ ${ }^{3}$ Servicio de Salud Mental, Hospital Clínico Universitario de Valencia, España. \\ ${ }^{4}$ CIBER Fisiopatología Obesidad y Nutrición (CIBERobn), Instituto Salud Carlos III, Madrid, España.
}

Group cognitive remediation and emotion skills training for patients with obesity. A pilot study

\begin{abstract}
Cognitive remediation and emotion skills training was initially designed by the Tchanturia group for individual intervention in patients with eating disorders. The purpose of the present study is to present its adaptation to patients with obesity in group format. It is structured in 8 weekly sessions that work on central executive functions, the relation between emotion and thought, and emotion regulation in oneself and others. In addition, we present the preliminary results of its application to a group of 5 participants with morbid obesity to assess its possible efficacy. The results indicate that, after the intervention, the patients showed improvement in their cognitive and emotional skills, as well as in their behaviors related to eating and weight. This program can be a useful complementary intervention in the initial stages of obesity treatment.
\end{abstract}

Keywords: CREST; cognitive remediation; emotion; eating disorders; obesity.

Resumen: El entrenamiento en remediación cognitiva y habilidades emocionales fue diseñado inicialmente por el grupo de Tchanturia para la intervención individual de pacientes con trastornos alimentarios. El objetivo del presente trabajo es exponer su adaptación a pacientes con obesidad en formato grupal. Se estructura en 8 sesiones semanales en las que se trabaja funciones de ejecutivo central, la relación entre emoción y pensamiento, y el manejo emocional en un mismo y en los demás. Además, presentamos los resultados preliminares de su aplicación a un grupo de 5 participantes con obesidad mórbida con el fin de valorar su posible eficacia. Los resultados indican que tras la intervención, las pacientes mostraron una mejoría en sus habilidades cognitivas y emocionales, así como en las conductas relacionadas con la alimentación y el peso. Este programa puede ser una intervención útil complemento del tratamiento de la obesidad en sus fases iniciales.

Palabras clave: CREST; remediación cognitiva; emoción, trastornos alimentarios, obesidad.

\section{Introducción}

La Organización Mundial de la Salud (OMS, 2016) define la obesidad y el sobrepeso como una acumula-

Recibido: 11 diciembre 2016; aceptado: 26 junio 2017 Correspondencia: Conxa Perpiñá, Universidad de Valencia, Av. Blasco Ibáñez 21, 46010 Valencia, España. Correo-e: perpinya@uv.es Agradecimientos: Este estudio ha sido subvencionado por el Ministerio de Ministerio de Economía y Competitividad por medio del proyecto de investigación (PSI2014-51920-R). CIBERobn es una iniciativa de ISCIII. ción anormal y excesiva de grasa perjudicial para la salud y que se manifiesta por un exceso de peso y volumen corporales. La obesidad se diagnostica si el índice de masa corporal (IMC) se sitúa en valores iguales o superiores a $30 \mathrm{Kg} / \mathrm{m}^{2}$, y se considera que el contenido de grasa en mujeres con obesidad es superior al $35 \%$ y en hombres es superior al 30\%. La prevalencia de esta enfermedad aumenta cada año en la mayoría de los países desarrollados, en España la última encuesta realizada por el Sistema Nacional de Salud (SNS; 2015) indicó que el $53.7 \%$ de los adultos padecen obesidad o sobre- 
peso, y que los porcentajes en población infanto-juvenil (entre 2 y 17 años) se sitúan en un 27.8\%. La obesidad es un factor de riesgo para múltiples enfermedades físicas como diabetes, hipertensión, enfermedad cardiovascular, apnea del sueño y varias formas de cáncer (Catalá y Gribés, 2010). Presenta una elevada comorbilidad psiquiátrica y una importante afectación en el funcionamiento de la vida diaria, así como un elevado coste económico a nivel social. En España, se estima que la obesidad origina el 7\% del gasto sanitario total (aproximadamente 2.500 millones de euros al año) (Catalá y Girbés, 2010).

El tratamiento eficaz para esta enfermedad implica una intervención multidisciplinar centrada en aspectos médicos, cognitivos y conductuales con el objetivo de modificar el estilo de vida y, en consecuencia, conseguir una reducción de peso (NICE, 2004). Entre los tratamientos considerados más eficaces se encuentra la terapia cognitivo-conductual combinada con dieta y ejercicio físico (Shaw, O'Rourke, Del Mar, y Kenardy, 2005). No obstante, los datos sobre su eficacia no son ideales, sobre todo en el mantenimiento de los resultados, ya que la mayoría recupera un $30-35 \%$ del peso perdido durante la intervención una vez finalizado el tratamiento (Wadden, Butryn, y Byrne, 2004; Cooper et al., 2010; Camacho-Laraña, Alcalá-Pérez, y Nieves-Alcalá, 2015).

Gran parte del interés de la investigación en obesidad se ha dirigido a estudiar qué otros aspectos pueden influir en su génesis y su mantenimiento. Por ejemplo, teniendo en cuenta la dificultad que estos pacientes presentan para inhibir la ingesta y frenar sus impulsos frente a los alimentos considerados «palatables» o apetitosos (Gionta, 1995) se ha llegado a conceptualizar la obesidad como una adicción a la comida (Gearhardt, Frilo, Dileone, Brownell, y Potenza, 2011). Otras investigaciones se han centrado en el estudio de las funciones ejecutivas y han demostrado que los pacientes con obesidad presentan problemas en la capacidad de tomar decisiones (Daniel, Stanton, y Epstein, 2013; Qavam, Anisan, Fathi, y Pourab, 2015), dificultades para demorar la gratificación a corto plazo a pesar de las consecuencias que puedan existir a largo plazo (Perpiñá, Segura, y Sánchez, 2017), y dificultades en la capacidad para planificar y solucionar problemas (Qavam et al., 2015). Todos estos déficits significan un obstáculo para la pérdida de peso y su mantenimiento, ya que cambiar los hábitos de ingesta y actividad implica, entre otros aspectos, ser capaz de cambiar las conductas en función de las consecuencias. Una nueva línea de investigación se está centrando en los aspectos emocionales y de cognición social en pacientes con obesidad. Los estudios todavía son escasos, aunque sus resultados muestran la presencia de dificultades en la capacidad para identificar, describir y expresar las emociones (Da Ros, Vinai, Gentile, Forza, y Cardetti, 2011; Giel et al., 2016), así como en la regulación emocional (Kittel, Brauhardt, y Hilbert, 2014). En concreto, los estudios plantean que cuando estos pacientes experimentan emociones desagradables suelen usar la conducta de comer para intentar reducirlas o suprimirlas (Leehr et al., 2015), lo cual repercute en su exceso de peso.

En la actualidad se entiende que la obesidad, los trastornos alimentarios y los comportamientos relacionados con las alteraciones del comer se ubican en un mismo continuo de trastornos relacionados con el peso, encontrándose en un extremo la anorexia nerviosa (AN) y en el otro la obesidad (Neumark-Sztainer et al., 2007). Además de compartir factores de riesgo biológicos y ambientales (Neumark-Sztainer et al., 2007), todos estos problemas pueden ocurrir en un mismo individuo simultanea o secuencialmente, o aumentar en gravedad a lo largo del tiempo (Neumark-Sztainer, Wall, Eisenberg, Story, y Hannan, 2006). Por otra parte, los nuevos hallazgos en la investigación sobre aspectos cognitivos, neuropsicológicos y emocionales apoyan una conceptualización integrada de los trastornos alimentarios y de la obesidad como problemas relacionados con el peso y la ingesta debido a que se han encontrado dificultades similares. Los pacientes con AN presentan una pobre flexibilidad cognitiva evidenciada en su dificultad para adecuar su comportamiento a las demandas ambientales (Tchanturia et al., 2012; Aloi et al., 2015); una débil coherencia central, que les lleva a ignorar los aspectos globales y salientes de una situación y centrarse en los detalles (Aloi et al., 2015; Weider, Indredavik, lydersen, y Hestad, 2016); y un proceso de toma de decisiones centrado en la búsqueda de gratificaciones a corto plazo ignorando las consecuencias a largo plazo (Galimberti et al., 2013; Matsumoto et al., 2015). En los pacientes con bulimia nerviosa y trastorno por atracón se ha observado un estilo cognitivo caracterizado por la inflexibilidad mental, perseverancia y problemas de cambio de enfoque (Ariza et al., 2012; Mobbs, Iglesias, Golay, y Van der Linden, 2011).

El grupo de investigación de Tchanturia desarrolló una adaptación de la terapia de remediación cognitiva (cognitive remediation therapy; CRT) para pacientes con trastornos alimentarios. Es una intervención protocolizada cuyo objetivo es que los pacientes aprendan nuevas estrategias y habilidades cognitivas para resolver problemas de todo tipo de un modo más eficaz (Tchanturia y Lock, 2011). Se lleva a cabo de manera complementaria a su tratamiento habitual, y les permite realizar 
un trabajo terapéutico sin centrarse en aspectos de la alimentación. Esta intervención ha mostrado ser eficaz para mejorar la flexibilidad cognitiva (Brockmeyer et al., 2013; Tchanturia, Lloyd, y Lang, 2013; Giombini, Moynihan, Turco, y Nesbitt, 2016), la coherencia central (Lock et al., 2013; Giombini et al., 2016), y la calidad de vida tras el tratamiento en pacientes con AN. Esta mejora se asocia a la reducción de la psicopatología alimentaria y la clínica de los pacientes (Lock et al, 2013; Dingemans et al., 2014). La CRT también se ha aplicado en pacientes con obesidad (CRT-O) (Raman, Hay, y Smith, 2014). En este caso, las habilidades cognitivas que se trabajan incluyen la flexibilidad cognitiva, la coherencia central (favoreciendo la atención al detalle) y la resolución de problemas. Actualmente, Smith y su equipo están realizando un estudio para evaluar la eficacia de la CRT-O, pero todavía no se han publicado resultados preliminares.

El grupo de Tchanturia posteriormente $\mathrm{y}$, atendiendo a las dificultades en la regulación emocional de las pacientes con trastornos alimentarios (Kittel et al., 2014; Caglar-Nazali et al., 2014), desarrolló el programa cognitive remediation and emotion skills training (CREST) (Davies et al., 2012), en el que, además de trabajar sobre las dificultades neuropsicológicas, también lo hace sobre los aspectos socio-emocionales. Hasta la fecha, los pocos estudios que han evaluado su eficacia están ofreciendo resultados esperanzadores en pacientes con AN (Davies et al., 2012; Harrison, Mountford, y Tchanturia, 2014). Específicamente, tras la intervención CREST mejora la flexibilidad cognitiva (Davies et al., 2012), la identificación y regulación emocional, y se reduce la sintomatología alimentaria (Money, Genders, Treasure, Schmidt, y Tchanturia, 2011).

Dado que los pacientes con obesidad exhiben dificultades tanto en sus funciones ejecutivas como en la regulación emocional, consideramos que el programa CREST puede mejorar la eficacia de los tratamientos de reducción de peso utilizados habitualmente. Hasta donde llega nuestro conocimiento, no existe ninguna aplicación del CREST en pacientes con obesidad, por lo que es importante investigar acerca de su eficacia en las alteraciones relacionadas con la obesidad. Por tanto, los objetivos del presente trabajo fueron: 1) describir la adaptación que hemos realizado del programa CREST para la intervención grupal de pacientes con obesidad (CREST-O) en el contexto sanitario público, con la finalidad de mejorar las capacidades cognitivas y emocionales alteradas en este tipo de pacientes; y 2) analizar los resultados preliminares de un estudio piloto sobre la eficacia del programa CREST-O en pacientes con obesidad.

\section{Método}

\section{Desarrollo de la CREST-O para el tratamiento de pacientes con obesidad}

Hemos realizado una adaptación del programa CREST para pacientes con obesidad en formato grupal. Además de las variables habituales en el estudio de la obesidad, se consideraron los déficits neuropsicológicos específicos en este tipo de pacientes. La adaptación se realizó considerando diversas limitaciones del SNS, como la falta de espacio y tiempo, y las largas listas de espera para recibir tratamiento psicológico. Estos aspectos generan la necesidad de realizar tratamientos eficientes en términos de coste/eficacia. Dado que la CREST-O es una intervención complementaria a los tratamientos habituales, se desarrolló un programa de intervención de baja intensidad, y se enfatizó el papel activo del paciente en sesión y mediante la realización de las tareas fuera de sesión.

El programa CREST-O es una intervención grupal protocolizada cuyo objetivo es entrenar algunas capacidades de funcionamiento ejecutivo y emocional e incorporarlas al funcionamiento cotidiano. Se pretende facilitar tanto la pérdida de peso como su mantenimiento, mediante la vinculación de los patrones alimentarios y de ejercicio físico a unos estilos de funcionamiento mejorados de pensamiento y emociones. El entrenamiento va dirigido a pacientes con obesidad que estén siguiendo su tratamiento médico-dietético habitual, acompañado o no de otras estrategias conductuales.

Se compone de cuatro módulos en los que se trabajan los siguientes aspectos: a) Funciones ejecutivas tales como la capacidad de coherencia central (atención al detalle), el cambio de enfoque y la habilidad para solucionar problemas, organizar y planificar; b) Relación entre pensamiento y emociones. Conocimiento de qué son y cómo funcionan las emociones, y cómo se relacionan con la forma de pensar; c) Reconocimiento emocional en uno mismo y en los demás, aumento de las experiencias positivas en el día a día y potenciación de las fortalezas personales; y d) Función de las emociones y el manejo emocional en uno mismo y con respecto a los demás. El programa se desarrolla a lo largo de 8 sesiones de una hora y media de duración y con frecuencia semanal. A excepción de la primera, en la que se explica el fundamento y objetivo de la intervención y se trabaja la cohesión grupal, cada sesión se inicia con el repaso de las tareas «para casa» recomendadas en la sesión anterior, se sigue con la psicoeducación de la habilidad concreta a trabajar en esa sesión, y de los ejercicios para practicar tal habilidad. Se finaliza siempre con la descripción de las tareas y cambios que tienen que realizar 
esa semana entre sesiones, hasta revisarse en la sesión siguiente. En la Tabla 1 se muestra la estructura y secuencia de los objetivos trabajados en cada una de las sesiones. En la Tabla 2 se describen las tareas utilizadas para el entrenamiento de las diferentes habilidades.

El estilo terapéutico ha de ser colaborativo, utilizando estrategias de motivación y refuerzo, siendo creativo en el desarrollo de las tareas, mostrándose interesado acerca de las experiencias de los pacientes mientras realizan los ejercicios, siendo positivo, e intentando reconducir el pensamiento negativo del paciente durante las sesiones.

\section{Participantes}

El grupo CREST-O estuvo formado inicialmente por 6 participantes, todas mujeres, que aceptaron voluntariamente participar en las sesiones de intervención. Ninguna de las pacientes presentaba ningún trastorno alimentario, ni ninguna otra comorbilidad psiquiátrica. En el transcurso de la intervención una de las pacientes abandonó la terapia por motivos de trabajo, por lo que finalmente fueron 5 personas las terminaron la intervención.
La participante $1(\mathrm{P} 1)$ tenía 30 años, pesaba $96.1 \mathrm{Kg}$ $(\mathrm{IMC}=42.09)$, estaba soltera y trabajaba como auxiliar de veterinaria. Había perdido peso en ocasiones anteriores, realizando distintas dietas y ejercicio físico, pero siempre había vuelto a engordar. En el momento de la intervención se encontraba realizando una dieta hipocalórica y estaba en seguimiento por su médico de atención primaria (MAP). Presentaba algunos episodios de picoteo y atracón en respuesta a desencadenantes internos (enfado, aburrimiento, ansiedad y tristeza).

La participante 2 (P2) tenía 61 años, pesaba $105 \mathrm{Kg}$ (IMC $=40.01$ ), convivía con su marido y su hijo (quien estaba diagnosticado de un trastorno mental grave), había realizado estudios universitarios pero actualmente era ama de casa. Se encontraba en tratamiento para la obesidad con su MAP. Estaba muy motivada para la pérdida de peso por diversos problemas de salud, pero no había conseguido llegar a un peso saludable. Ella atribuía el fracaso de los tratamientos a que tenía episodios de picoteo ante desencadenantes internos y externos que no conseguía controlar.

La participante 3 (P3) tenía 31 años, pesaba $118 \mathrm{Kg}$ $(\mathrm{IMC}=42.09)$, estaba soltera y se encontraba desem-

Tabla 1. Objetivos terapéuticos abordados en cada sesión.

\begin{tabular}{|c|c|}
\hline Sesión & Objetivos terapéuticos \\
\hline 1 & $\begin{array}{l}\text { - Establecer la relación terapéutica y fomentar la cohesión grupal. } \\
\text { - Presentación del programa CREST. } \\
\text { - Aumentar la conciencia sobre los estilos de pensamiento. } \\
\text { - Relacionar los estilos de pensamiento con la vida cotidiana. } \\
\text { - Trabajar el cambio de enfoque. } \\
\text { - Trabajar la capacidad para atender al detalle. }\end{array}$ \\
\hline 2 & $\begin{array}{l}\text { - Favorecer el cambio de enfoque. } \\
\text { - Entrenar habilidades de solución de problemas, organización y planificación. }\end{array}$ \\
\hline 3 & $\begin{array}{l}\text { - Reflexionar sobre los estilos de pensamiento y sobre lo que los pacientes han aprendido sobre su forma de pensar } \\
\text { - Aprender qué son las emociones. } \\
\text { - Relacionar el estilo de pensamiento y la emoción con la vida diaria. }\end{array}$ \\
\hline 4 & $\begin{array}{l}\text { - Favorecer la identificación y el reconocimiento de las emociones propias. } \\
\text { - Apreciar las emociones como algo transitorio y temporal. } \\
\text { - Aumentar las experiencias positivas en su vida diaria. }\end{array}$ \\
\hline 5 & $\begin{array}{l}\text { - Aumentar las experiencias positivas en su vida diaria. } \\
\text { - Identificar fortalezas propias. }\end{array}$ \\
\hline 6 & $\begin{array}{l}\text { - Entender la utilidad de las emociones propias. } \\
\text { - Aumentar la conciencia sobre la variabilidad de las emociones. } \\
\text { - Entrenar en el manejo de emociones propias. }\end{array}$ \\
\hline 7 & $\begin{array}{l}\text { - Continuar con el entrenamiento en el manejo de emociones propias. } \\
\text { - Entender la utilidad de las emociones de los demás. } \\
\text { - Mejorar la comunicación de las propias emociones a los demás. }\end{array}$ \\
\hline 8 & $\begin{array}{l}\text { - Practicar la habilidad de asertividad. } \\
\text { - Entrenar en el reconocimiento de las emociones de los demás. } \\
\text { - Favorecer la terminación de la terapia. }\end{array}$ \\
\hline
\end{tabular}


Tabla 2. Descripción de las tareas y sus correspondientes objetivos para cada habilidad entrenada en las sesiones.

TAREA OBJETIVO

Atención al detalle

Un buen resumen Estimular la capacidad de atender a los detalles, animando a los pacientes a analizar toda la información del texto y centrarse en los detalles relevantes e irrelevantes.

Figuras complejas Describir a su compañero una figura compleja para que sea capaz de representarla, atendiendo especialmente a los detalles.

Cambio de enfoque

Dos tareas a la vez Practicar la capacidad de utilizar paralelamente el pensamiento global y la atención a los detalles mediante la identificación de determinadas categorías de información relevante entre una cantidad mayor de información irrelevante.

STROOP Practicar la alternancia entre diferentes aspectos de un estímulo o entre diferentes reglas para realizar una actividad, de forma rápida y con exactitud utilizando la tarea STROOP con imágenes, números y palabras.

Ilusiones Practicar mantener en la mente dos ideas a la vez y alternar entre diferentes piezas de información mediante el análisis de ilusiones ópticas.

Solución de problemas, planificación y organización

Planificación Estimular esta capacidad mediante la planificación y organización de situaciones cotidianas.

Solucionando Solucionar problemas que se presentan típicamente en pacientes con obesidad.

problemas

Entender qué son las emociones y cómo funcionan

Clasificando

emociones

Fomentar la identificación de las emociones y la distinción entre emociones positivas y negativas mediante la

Emociones y

pensamiento

clasificación de palabras emocionales.

El ciclo del procesamiento emocional

Aprender la relación entre las emociones y el pensamiento explorando de un modo sencillo cómo interactúan y planteando ejemplos de su propia vida.

Aprender el ciclo del procesamiento emocional e identificar en qué partes del ciclo es más fácil hacer cambios para regular la emoción mediante el completamiento de un ciclo desde el inicio hasta el final.

Aumentar la conciencia sobre las diferencias individuales en las respuestas de los individuos ante la misma información mediante la discusión sobre el ejercicio.

Las emociones en Aprender cómo las emociones se asocian con sensaciones fisiológicas situándolas en el cuerpo.

nuestro cuerpo

Cuestionario

Aumentar la conciencia y comprensión de los pacientes sobre su estilo emocional y de pensamiento mediante la emocional realización del cuestionario de autoinforme.

\section{Identificar y describir emociones}

Lista de palabras emocionales

Describiendo emociones

Cambiando escenarios

Facilitar el reconocimiento de emociones presentes en uno mismo, identificando y etiquetando las emociones más relevantes para cada uno,

Favorecer la identificación y el reconocimiento de emociones difíciles mediante el uso de nuevas estrategias.

Animar a los pacientes a ver las emociones como algo transitorio y temporal a través de la lectura de varias situaciones en las que se experimentan diferentes emociones.

Aumentar las experiencias positivas

Encontrando Reconocer e identificar aspectos positivos en uno mismo y en el mundo, mediante el uso de diversas estrategias, estrategias y elegir alguna de estas estrategias con el objetivo de amplificar las experiencias positivas.

Reforzar las fortalezas personales

Lista de fortalezas Identificar las fortalezas personales, centrándose en los aspectos que cada uno valora de sí mismo, y reforzarlas. personales

Entender la utilidad de las emociones en uno mismo y en los demás

Lo que pueden

hacer las emociones
Entender la función de las emociones centrándose en qué cosas nos comunican sobre nosotros mismos y sobre nuestro medio y en cómo nos pueden ayudar a comunicarnos con los demás. Facilitar la comunicación de necesidades. 
Tabla 2. Descripción de las tareas y sus correspondientes objetivos para cada habilidad entrenada en las sesiones. (Continuación) TAREA OBJETIVO

Ser consciente de la variabilidad de las emociones

Mapa lingüístico de Ayudar a ver cómo las emociones varían en fuerza e intensidad, buscando emociones intensas y emociones reemociones lacionadas de menor intensidad.

El termómetro Favorecer la consciencia de la variabilidad emocional, centrándose en los cambios de intensidad de una o varias emocional emociones y relacionándolo con los cambios en la intensidad de la respuesta fisiológica y del cambio comportamental.

Manejo de emociones

Manejando

Aumentar la consciencia sobre cómo gestionar las emociones difíciles explorando las ventajas y desventajas de emociones difíciles

las estrategias que utilizan habitualmente.

La jirafa rosa

Entender que la evitación, como estrategia de afrontamiento emocional, puede ser contraproducente mediante el uso de un experimento.

Comunicación de las emociones y necesidades propias

¿Cómo expresar lo Identificar su forma de comunicar las emociones y necesidades mediante la auto-observación de su conducta en que sientes y

necesitas?

Siendo asertivo Identificar estilos de conducta interpersonal reflexionando sobre cómo suelen responder habitualmente y como se sienten en diferentes situaciones.

¿Qué podría hacer Aprender a comunicar los sentimientos y necesidades mediante la práctica de la asertividad.

mejor?

Reconocimiento emocional en los demás

Expresión facial Entender la importancia de la expresión facial para comprender las emociones y aprender claves para interpretarlas mediante la identificación de lo que están sintiendo diferentes personas a través de su expresión facial.

pleada y estudiando un máster universitario. Refería haber realizado muchas dietas previas pero no había conseguido sus objetivos. Presentaba episodios de picoteo y de atracón subjetivos en respuesta al aburrimiento y a estar en presencia de comida que consideraba «prohibida». En el momento de la intervención se encontraba en seguimiento por su MAP.

La participante 4 (P4) tenía 49 años, pesaba $115 \mathrm{Kg}$ $(\mathrm{IMC}=42.27)$, convivía con su marido y sus dos hijos, y tenía un negocio familiar. Presentaba sintomatología ansiosa leve reactiva a diversas circunstancias familiares que le dificultaba la pérdida de peso debido a que utilizaba la comida como estrategia de afrontamiento. Presentaba un patrón alimentario desestructurado por los horarios laborales y las cargas familiares, y episodios de picoteo y atracón en respuesta a diversas emociones desagradables y en presencia de comida «prohibida». Describía que nunca había tenido problemas de peso hasta hace varios años en los que había engordado debido a este patrón alimentario y a la menopausia. Actualmente estaba en seguimiento por su MAP para el tratamiento de la obesidad.

La participante 5 (P5) tenía 51 años, pesaba $106 \mathrm{Kg}$ (IMC $=37.19)$, convivía con su marido y sus dos hijas, y era profesora de francés. Comentó que varios problemas de salud la habían motivado para perder peso pero que, a pesar del tratamiento con el MAP, no lo conseguía. Presentaba episodios de picoteo en respuesta a desencadenantes externos.

\section{Instrumentos}

Medidas antropométricas. Tomamos medidas del peso y la altura de cada participante antes y después de la intervención para el cálculo de su IMC. Los pesos que se refieren en la presentación de las participantes son de la evaluación previa a iniciarse la primera sesión de la CREST-O.

Juego de azar de Iowa (The Iowa gambling task, IGT; Bechara, Damasio, Damasio, y Anderson, 1994). Prueba neuropsicológica que evalúa la capacidad para tomar de decisiones de riesgo simulando decisiones que se toman en la vida cotidiana. La tarea consiste en elegir cartas de 4 barajas con el objetivo de intentar ganar el máximo dinero posible. Cada elección implica la ganancia o pérdida de dinero. Hay 2 barajas (A y B) de alto riesgo que proporcionan mucho dinero a corto plazo pero también muchas pérdidas a largo plazo, y 2 barajas (C y D) de 
bajo riesgo que proporcionan pocas ganancias a corto plazo, pero pocas pérdidas a largo plazo. La puntuación total se calcula con la fórmula $(\mathrm{C}+\mathrm{D})-(\mathrm{A}+\mathrm{B})$ tras 100 elecciones.

Test de Clasificación de Cartas de Wisconsin (Wisconsin card sorting test, WSCT, Grant y Berg, 1948). Evalúa la capacidad para cambiar las estrategias cognitivas en respuesta a las modificaciones ambientales. En este caso utilizamos la versión para ordenador de aplicación individual, formada por 4 tarjetas estímulo y 128 tarjetas respuesta. La tarea consiste en clasificar las tarjetas respuesta en una de las tarjetas estímulo según un criterio (color, forma o número) desconocido para el evaluado. El criterio de clasificación cambia cada 10 emparejamientos consecutivos correctos. La prueba termina al completar 6 categorías correctamente o cuando se han presentado 128 tarjetas respuesta. En este estudio las variables a estudiar son: Total de categorías completas, número de errores totales y número de errores perseverativos.

Test de las Figuras Incrustadas (Embedded figures test group, GEFT, Witkin, Oltman, Raskin, y Karp, 1971). Evalúa la tendencia a centrarse en el detalle o atender al conjunto de la información (coherencia central). Se utilizó la versión grupal aplicada individualmente, esta versión incluye 25 figuras complejas en la que se debe distinguir una figura simple. La prueba incluye una sección de práctica ( 7 figuras) y dos secciones de test ( 9 figuras cada una) con un límite de tiempo de 5 minutos cada una. La puntuación total es la suma de las puntuaciones en las secciones 2 y 3 .

Escala de Alexitimia de Toronto (Toronto Alexithymia Scale, TAS-20, Bagby, Parker, y Taylor, 1994; versión en español de Sandín, Chorot, Santed y Jimenez, 1996). Autoinforme de 20 ítems en los que se valora el grado de acuerdo mediante una escala Likert de 5 puntos $(1$ «Nada de acuerdo» a 5 «Totalmente de acuerdo»). Se compone de tres factores: dificultad para identificar sentimientos y diferenciarlos de las sensaciones corporales o fisiológicas que acompañan la activación emocional; dificultad para describir los sentimientos a los demás; y un estilo de pensamiento orientado hacia lo externo. La adaptación española (Sandín et al., 1996), obtuvo una adecuada fiabilidad $($ alfa $=.81)$ y validez.

Cuestionario de Afecto Positivo y Negativo (Positive and Negative Affect Schedule, PANAS, Watson, Clark y Tellegen, 1988; Versión en español de Sandín et al., 1999). Autoinforme de 20 ítems para la evaluación del afecto positivo y negativo. La escala de afecto positivo (PANAS-AFP) evalúa la capacidad de involucrarse en actividades placenteras. La escala de afecto negativo (PANAS-AFN) evalúa la presencia de estrés percibido. La adaptación española (Sandín et al., 1999) mostró una elevada consistencia interna en las dos escalas (alfa $=$ $.89-.91)$.

Medida de Apetito Situacional (Situational Appetite Measures, SAM, Stanton, García, y Green, 1990). Es un cuestionario para la evaluación de los impulsos relacionados con la comida a través de 30 ítems en escala tipo Likert. Incluye una escala para evaluar la presencia de impulsos por comer (SAM-U) y una para evaluar la eficacia del autocontrol de los impulsos (SAM-E). El SAM ha demostrado valores altos de fiabilidad (alfa $>.70$ ), estabilidad (fiabilidad test-retest $>.70$ ) y validez divergente frente a medidas de estado de ánimo (Stanton et al., 1990). En este estudio se usó una versión experimental de este instrumento.

Variables relevantes del problema. Se utilizó un cuestionario creado ad hoc con 7 ítems en escala tipo Likert (0-nada a 9-muchísimo) para evaluar medidas relevantes relacionadas con la obesidad antes y después del tratamiento, tales como número de comidas al día; presencia y frecuencia de episodios de picoteo y atracones, y sus desencadenantes; presencia e intensidad de conductas de evitación; control sobre la comida y el peso; y estado de ánimo.

Evaluación cualitativa. La evaluación cualitativa se realizó mediante un cuestionario de preguntas abiertas y respuesta libre en las que las participantes describen qué han aprendido, qué aspectos les han resultado útiles y/o innecesarios, y qué creen que se podría mejorar en el programa CREST-O.

Satisfacción con el programa. Se utilizó una escala de opinión sobre el tratamiento (adaptada de Borkovec y Nau, 1972) para valorar la satisfacción con el programa CREST-O. Esta escala se compone de 6 ítems, valorados en una escala Likert de 10 puntos (0-nada, 10-muchísimo), que evalúan en qué medida el programa les ha resultado lógico, útil en su caso y útil para otros problemas psicológicos, en qué medida lo recomendarían a un amigo, si les ha resultado aversivo o desagradable y en qué grado les ha satisfecho.

Calidad de vida. Índice de calidad de vida (ICV, Mezzich et al., 2000). Es un autoinforme formado por 10 ítems que evalúan el bienestar físico y psicológico, el autocuidado y funcionamiento independiente, el funcionamiento ocupacional y el funcionamiento interpersonal, el apoyo social-emocional, apoyo comunitario, la plenitud personal, la satisfacción espiritual y la calidad de vida global. Cada uno de los ítems se valora en una escala Likert de 10 puntos siendo 1 malo y 10 excelente.

\section{Procedimiento}

Este proyecto fue realizado según la declaración de Helsinki y aprobado por el comité de ética del hospital 
universitario de la Ribera (Alzira, Valencia). Se incluyó a mujeres con edad entre 18 y 65 años, con diagnóstico de obesidad que estaban siendo tratadas en el SNS. Se incluyó a mujeres que no habían sido sometidas a ningún tipo de cirugía bariátrica, no cumplían criterios diagnósticos para ningún trastorno alimentario y no presentaban ninguna enfermedad física y/o mental grave ni un déficit cognitivo y/o sensoperceptivo que interfiriese en el desarrollo de la intervención. La intervención se llevó a cabo por una psicóloga clínica formada en la aplicación del CREST-O. Antes y después del desarrollo del programa se administraron las pruebas neurospicológicas y los cuestionarios antes mencionados. En la última sesión se administró un cuestionario formado por diversas preguntas abiertas para valorar cualitativamente las opiniones de las participantes respecto a la eficacia de la terapia. Todas las participantes firmaron el consentimiento informado para participar en el programa CREST-O.

\section{Resultados}

\section{Medidas antropométricas}

En dos de las participantes (P1, P3) se observó una pérdida de peso significativa entre $4 \mathrm{Kg}$ y $7 \mathrm{Kg}$.

\section{Variables neuropsicológicas}

Las puntuaciones directas de cada participante se muestran en la Tabla 3. Más de la mitad de las participantes mejoraron el rendimiento en el IGT tras la intervención $\left(M_{\mathrm{PRE}}=4 ; M_{\mathrm{POST}}=27.6\right)$. En la evolución de las pacientes se aprecia que la mayor parte de ellas pasa de puntuaciones negativas a positivas, lo cual significa una mejora en su toma de decisiones en el sentido de equilibrar las elecciones arriesgadas. La puntuación de corte en esta prueba se establece en 10 (Bechara et al., 2001). En el WCST, se observa en la mayoría de las participantes una reducción en el número de errores totales $\left(M_{\mathrm{PRE}}=\right.$ $\left.40.8 ; M_{\mathrm{POST}}=25.8\right)$ y el de errores perseverativos $\left(M_{\mathrm{PRE}}=\right.$ 29.4; $M_{\text {POST }}=22$ ), así como un aumento en el número de categorías completadas correctamente $\left(M_{\mathrm{PRE}}=4.6 ; M_{\mathrm{POST}}\right.$ $=5$ ). La tasa de errores disminuye, aunque por debajo de puntuaciones normativas, pero el número de categorías completadas correctamente se sitúa en torno a 5, es decir puntuación normativa (Grant y Berg, 1948; Periáñez y Barceló, 2001). En el GEFT, todas las participantes mejoraron su puntuación después de la intervención $\left(M_{\mathrm{PRE}}=\right.$ 10.4; $\left.M_{\text {POST }}=13.4\right)$. Dado que la puntuación máxima en esta tarea es 18, la media de la ejecución antes de la intervención se situaba en el segundo cuartil, pero pasa al ter- cer cuartil después de la intervención; incluso 2 de las pacientes obtendrían puntuaciones de cuarto cuartil (Witkin et al., 1971).

\section{Variables de procesamiento emocional}

Todas las participantes mostraron una reducción de la puntuación TAS-20 total $\left(M_{\mathrm{PRE}}=49,6 ; M_{\mathrm{POST}}=44.4\right)$, siendo más relevante en P1, P2 y P4. Se observa también una disminución de las puntuaciones en la escala de identificación de sentimientos $\left(M_{\mathrm{PRE}}=15 ; M_{\mathrm{POST}}=14.2\right)$, la escala de descripción de sentimientos $\left(M_{\mathrm{PRE}}=12.2\right.$; $\left.M_{\text {POST }}=12\right)$ y la escala de orientación de pensamiento $\left(M_{\mathrm{PRE}}=22.4 ; M_{\mathrm{POST}}=18.2\right)$. En el cuestionario PANAS, la mayoría de las participantes se mantuvieron estables o mejoraron tanto el afecto positivo $\left(M_{\mathrm{PRE}}=28.6 ; M_{\mathrm{POST}}=29.6\right)$ como el afecto negativo $\left(M_{\mathrm{PRE}}=27.2 ; M_{\mathrm{POST}}=26.4\right)$.

\section{Variables de psicopatología alimentaria}

En cuanto al cuestionario SAM, se observa un leve aumento del impulso por comer en la mayoría de las participantes $\left(M_{\mathrm{PRE}}=3.23 ; M_{\mathrm{POST}}=4.3\right)$, sin embargo todas aumentaron la expectativa de control de la ingesta tras la intervención $\left(M_{\mathrm{PRE}}=2.47 ; M_{\mathrm{POST}}=3.23\right)$.

\section{Variables relevantes del problema}

La mayoría de participantes presentaban un patrón alimentario irregular antes de la intervención, realizaban entre dos y tres comidas al día, y en algunos casos presentaban también ingestas después de cenar. Tras la intervención, todas las participantes realizaban 5 comidas al día (desayuno, media mañana, comida, merienda y cena) y solo P3 refirió realizar alguna ingesta después de cenar. Por otra parte, antes de la intervención P1, P3 y $\mathrm{P} 4$ refirieron haber presentado episodios de atracón subjetivos, con una frecuencia semanal de entre 1 y 2 veces, $\mathrm{P} 1$ además presentaba algún episodio de atracón objetivo. Estos episodios aparecían sobre todo frente a desencadenantes internos (aburrimiento y emociones desagradables) y en algún caso externo (ver comida prohibida). Todas las participantes presentaban episodios de picoteo. Tras la intervención las participantes refirieron seguir presentando algunos episodios de picoteo pero ninguna realizaba episodios de atracón objetivos ni subjetivos.

Antes de la intervención, las participantes evitaban algunas situaciones (como salir a cenar, salir con los amigos, pesarse, comer delante de la gente, cocinar para su marido e hijos y hacer deporte). El grado de evitación de estas conductas variaba de unas participantes a otras. 
Tabla 3. Resultados PRE/POST en las pruebas neuropsicológicas, en las variables de procesamiento emocional y en las variables de psicopatología alimentaria

\begin{tabular}{|c|c|c|c|c|c|c|c|c|c|c|c|c|c|c|c|c|c|c|c|c|c|c|c|c|c|c|}
\hline & \multicolumn{2}{|c|}{$\begin{array}{c}\text { IG } \\
\text { TOTAL }\end{array}$} & \multicolumn{2}{|c|}{$\begin{array}{l}\text { WCST } \\
\text { ERR. }\end{array}$} & \multicolumn{2}{|c|}{$\begin{array}{c}\text { WCST } \\
\text { ERR.PER }\end{array}$} & \multicolumn{2}{|c|}{$\begin{array}{l}\text { WCST } \\
\text { CAT }\end{array}$} & \multicolumn{2}{|c|}{$\begin{array}{c}\text { GEFT } \\
\text { AC. }\end{array}$} & \multicolumn{2}{|c|}{$\begin{array}{c}\text { TAS } \\
\text { F1 }\end{array}$} & \multicolumn{2}{|c|}{$\begin{array}{c}\text { TAS } \\
\text { F2 }\end{array}$} & \multicolumn{2}{|c|}{$\begin{array}{c}\text { TAS } \\
\text { F3 }\end{array}$} & \multicolumn{2}{|c|}{$\begin{array}{l}\text { TAS } \\
\text { TOT }\end{array}$} & \multicolumn{2}{|c|}{$\begin{array}{c}\text { PANAS. } \\
\text { AFP }\end{array}$} & \multicolumn{2}{|c|}{$\begin{array}{c}\text { PANAS. } \\
\text { AFN }\end{array}$} & \multicolumn{2}{|c|}{$\begin{array}{c}\text { SAM. } \\
\text { U }\end{array}$} & \multicolumn{2}{|c|}{$\begin{array}{l}\text { SAM. } \\
\text { E }\end{array}$} \\
\hline & PR & PS & PR & $\mathrm{P}$ & PR & $\mathrm{P}$ & $\mathrm{PR}$ & PS & PR & PS & PR & $\mathrm{P}$ & PR & PS & PR & PS & PR & PS & PR & PS & PR & PS & PR & PS & PR & PS \\
\hline P1 & -10 & 50 & 88 & 00 & 88 & 88 & 1 & 1 & 10 & 12 & 12 & 1 & 12 & 13 & 26 & 14 & 50 & 42 & 33 & $J_{1}$ & 26 & 25 & 7.3 & 15.7 & 13 & 16.6 \\
\hline P2 & 52 & 24 & 5 & 15 & 24 & 7 & 5 & 6 & 9 & 15 & 10 & 8 & 12 & 11 & 19 & 16 & 41 & 35 & 24 & 25 & 2 & 30 & 13 & 13 & 12.5 & 13.1 \\
\hline P3 & -28 & 64 & 1 & 8 & 9 & 5 & 6 & 6 & 13 & 14 & 19 & 18 & 1 & 15 & 24 & 1 & 56 & 52 & 3 & 31 & 2 & 26 & 19.7 & 16.5 & 11.3 & 13 \\
\hline P4 & -40 & 34 & 11 & 6 & 6 & 4 & 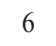 & 0 & 15 & 16 & 27 & 23 & 18 & 16 & 25 & 24 & 7 & 63 & 32 & 25 & 39 & 19 & 21.8 & 20.8 & 8.6 & 11.5 \\
\hline P5 & 46 & 28 & 41 & 12 & 20 & 6 & 5 & 6 & 5 & 10 & 7 & 7 & 6 & 5 & 18 & 18 & 31 & 30 & 21 & 32 & 19 & 32 & 9.2 & 9.5 & 16.1 & 21.5 \\
\hline
\end{tabular}

Nota: PR: Pre-intervención. PS: Post-intervención. P1: Participante 1. P2: Participante 2. P3: Participante 3. P4: Participante 4. P5: Participante 5. IGT: Iowa Gambling Task (Bechara et al., 1994). WCST: Wisconsin Card Sorting Test (Grant y Berg, 1948). WCST ERR: Errores totales. WCST ERR.PER: errores perseverativos. WCST CAT: categorías completadas. GEFT AC.: Group embedded Figures Test. Aciertos totales (Witkin et al., 1971). TAS: Escala de alexitimia de Toronto (Bagby et al., 1994). TAS F1: Alexitimia Dificultad para identificar sentimientos; TAS F2: Alexitimia Dificultad para describir sentimientos; TAS F3: Alexitimia Orientación externa del pensamiento. TAS TOT: Alexitimia Puntuación total. PANAS: Escala de afecto positivo y negativo- Versión española (Sandín et al., 1999). PANAS.AFP: Afecto positivo. PANAS.AFN: Afecto negativo. SAM: Medida de apetito situacional-Situational appetite measure (Stanton et al., 1990). SAM.U: Situational appetite measure urge. SAM.E: Situational appetite measure efficacy.

P1 era la que más situaciones y con mayor intensidad (5) evitaba, y las demás presentaban niveles de evitación intermedio (3-4). Tras la intervención, las conductas de evitación se redujeron en todos los casos.

El control percibido sobre la comida, el peso y otros aspectos de su vida aumentó en todas las participantes después de la intervención. Y el estado de ánimo percibido también mejoró en todos los casos $\left(M_{\mathrm{PRE}}=5\right.$; $\left.M_{\text {POST }}=1.6\right)$.

\section{Evaluación cualitativa}

Las participantes valoraron positivamente el programa CREST-O y consideran que satisfizo sus expectativas iniciales, refieren que les resultó efectivo y útil, que les ha ayudado a ser más conscientes de su problema y que les proporcionó habilidades para utilizar en la vida cotidiana como identificar sus emociones y manejarlas adecuadamente sin recurrir a la comida para sentirse mejor. Como aspectos a mejorar, la mayoría plantean que les gustaría que las sesiones tuviesen mayor duración y que el tratamiento fuese más largo.

\section{Satisfacción con el programa}

Las participantes valoraron que el programa CREST-O les pareció lógico $(M=8)$ y muy útil en su caso $(M=8.6)$, que lo recomendarían a un amigo que tuviese el mismo problema $(M=8.4)$ y que en su opinión sería útil para otros problemas psicológicos $(M=6.8)$. No les resultó en absoluto aversivo o desagradable $(M=0)$ y sí muy satisfactorio $(M=8.6)$.

\section{Calidad de vida}

Todas las participantes mostraron una mejoría en la percepción de su calidad de vida tras la intervención con el programa CREST-O, la media de la puntuación de calidad de vida percibida antes de la intervención fue de 4.4 y después fue de 7 .

\section{Discusión}

Las dificultades habidas en los tratamientos habituales de pérdida de peso en pacientes con obesidad hacen patente la necesidad de profundizar los factores implicados en el desarrollo y el mantenimiento de esta enfermedad. Los déficits en la toma de decisiones, la inflexibilidad cognitiva, la falta de atención al detalle y las dificultades en la planificación y solución de problemas (Qavam et al., 2015), junto a las dificultades emocionales para identificar, describir, expresar y manejar las emociones propias y de los demás (Giel et al, 2016; Kittel et al., 2014), podrían explicar parte de los problemas encontrados en el tratamiento de los pacientes con obesidad, sobre todo en el mantenimiento del peso perdido una vez acabado el tratamiento.

En este estudio nos planteamos que el programa CREST-O puede resultar de utilidad para mejorar la eficacia de los tratamientos habituales de reducción de peso en este tipo de pacientes, por lo que el objetivo de este trabajo ha sido desarrollar una adaptación del CREST (CREST-O) para la aplicación en grupo a pacientes con obesidad en el contexto sanitario público y valorar su eficacia mediante un estudio piloto con un 
grupo de pacientes con obesidad mórbida. El propósito del CREST-O es mejorar las funciones de flexibilidad cognitiva, el procesamiento global y la toma de decisiones reflexiva, la identificación de sentimientos y la regulación emocional, y relacionar todo ello con la ingesta de alimentos y la realización de ejercicio físico en su día a día. Gracias a este trabajo cognitivo y emocional, el programa contribuirá a que los pacientes pierdan el peso necesario mediante su tratamiento habitual, y mantengan el peso perdido una vez finalizado el tratamiento.

Los resultados de este estudio piloto han sido esperanzadores. Tras la intervención el proceso de toma de decisiones de las pacientes fue más reflexivo y centrado en las consecuencias a largo plazo, presentaron menos dificultades para cambiar de enfoque y más flexibilidad cognitiva, y su tendencia a la integración global para procesar la información fue menor consiguiendo centrarse más en los detalles de las diferentes situaciones. Asimismo, se produjo una mejoría en el procesamiento emocional, sobretodo en la alexitimia. Tras la intervención, la mayoría de las participantes mostraron menos dificultades para identificar y describir sentimientos, y menor orientación del pensamiento hacia lo externo. En cuanto a la psicopatología alimentaria, las participantes siguieron refiriendo impulsos de comer; no obstante, la percepción de control para llevar a cabo la conducta de ingesta aumentó. Además, todas las participantes valoraron positivamente el programa y se mostraron satisfechas con la intervención, afirmando que les había resultado muy útil para su problema.

Nuestros resultados son muy preliminares y no podemos llegar a conclusiones generales ni generalizables. Se trata de un estudio piloto realizado con solo 5 participantes, todas mujeres, y no hemos contado con un grupo control con el que poder contrastar los resultados. Por otra parte, tampoco podemos comparar los resultados con una muestra normativa de referencia completamente adecuada a las características sociodemográficas de nuestras participantes, ni contamos con baremos específicos de ejecución en población obesa, aunque ya empiezan a haber estudios que facilitan la ejecución media en estas pruebas en este tipo de pacientes, siendo semejante a lo que aquí hemos encontrado (Fagundo et al., 2012; Perpiñá et al., 2017). Otra de las limitaciones ha sido no haber podido recoger medidas intermedias, lo cual nos hubiera permitido contar con un diseño de línea base múltiple que hubiera dado más fortaleza a los datos obtenidos. Por último, la imposibilidad de contar con un diseño de tratamiento habitual con y sin CREST-O nos impide ofrecer resultados sobre su eficacia diferencial. Sin embargo, el paralelismo de lo acontecido con las pacientes con trastornos alimentarios quienes después de su entrenamiento en la CREST mejoraron en flexibilidad cognitiva, en identificación y regulación emocional (Davies et al., 2012) e incluso en sintomatología alimentaria (Money, Genders et al., 2011), además de que informaron de que las habilidades entrenadas les resultaron de utilidad en su cotidianidad (Money, Davies, y Tchanturia, 2011), nos hace confiar en el desarrollo de esta iniciativa terapéutica dado que estos resultados son los que hemos encontrado con nuestros pacientes con obesidad. Estos datos, junto con los resultados acerca de su eficacia, podrían hacer del programa CREST-O una intervención de elección en las fases iniciales del tratamiento (Money, Davies et al., 2011), facilitando que los pacientes se adhiriesen más fácilmente a la intervención habitual sobre la psicopatología alimentaria.

\section{Conflictos de intereses}

Los autores declaran que no existen conflictos de intereses

\section{Referencias}

Aloi, M., Rania, M., Caroleo, M., Bruni, A., Palmieri, A., Cauteruccio, M. A.,...Segura, C. (2015). Decision making, central coherence and set-shifting: a comparison between binge eating disorder, anorexia nervosa and healthy controls. $B M C$ Psychiatry, 15 . doi: 10.1186/s12888-015-0395-Z

Ariza, M., Garolera, M., Jurado, M. A., Garcia-Garcia, I., Hernan, I., Sanchez-Garre, C.,... Naberhaus, A. (2012). Dopamine genes (DRD2/ANKK1-TaqA1 and DRD4-7R) and executive function: their interaction with obesity. PLOS One, 7, e41482. doi: 10.1371/journal.pone.0041482

Bagby, R., Parker, J., \& Taylor, G. (1994). The twenty-item Toronto alexithymia scale-I. Item selection and cross-validation of the factor structure. Journal of Psychosomatic Research, 38, 23-32.

Bechara, A., Damasio, A. R., Damasio, H., \& Anderson, S. W. (1994). Insensitivity to future consequences following damage to human prefrontal cortex. Cognition, 50, 7-15.

Bechara, A., Dolan, S., Denburg, N., Hindes, A., Anderson, S. W., \& Nathan, P. E. (2001). Decision-making deficits, linked to a dysfunctional ventromedial prefrontal cortex, revealed in alcohol and stimulant abusers. Neuropsychologia, 39, 376-389. doi: 10.1016/S0028-3932(00)00136-6

Borkovec, T. D. y Nau, S. D. (1972). Credibility of analogue therapy rationales. Journal of Behavior Therapy and Experimental Psychiatry, 3, 257-260.

Brockmeyer, T., Ingenerf, K., Walther, S., Wild, B., Hartmann, M., Herzog, W.,... Friederich, H. (2013). Training cognitive flexibility in patients with anorexia nervosa: A pilot randomized controlled trial of cognitive remediation therapy. International Journal of Eating Disorders, 47, 24-31. doi: 10.1002/eat.22206

Caglar-Nazali, N. P., Corfield, F., Cardi, V., Ambwani, S., Leppanen, J., Olabintan, O.,... Treasure, J. (2014). A systematic review and meta-analysis of 'systems for social processes' in 
eating disorders. Neuroscience \& Biobehavioral Reviews, 42, 55-92. doi: 10.1016/j.neubiorev.2013.12.002

Camacho-Laraña, M., Alcalá-Pérez, V., y Nieves-Alcalá, S. (2015). Diferencias de género en pacientes con obesidad mórbida tributarios de cirugía bariátrica. Revista de Psicopatología y Psicología Clínica, 20, 189-198. doi: 10.5944/rppc. vol.20.num.3.2015.15892

Catalá, M., y Girbés, J. (2010). Estudio Valencia (II): Prevalencia de obesidad, hipertensión arterial, dislipemia, tabaquismo y resistencia a la insulina. Valencia: Generalitat. Conselleria de Sanitat.

Cooper, Z., Doll, H., Hawker, D., Byrne, S., Bonner, G., Eeley, E.,... Faiburn, C. (2010). Testing a new cognitive behavioural treatment for obesity: a randomized controlled trial with three-year follow-up. Behaviour Research and Therapy, 48, 706-713. doi: 10.1016/j.brat.2010.03.008

Da Ros, A., Vinai, P., Gentile, N., Forza, G., \& Cardetti, S. (2011). Evaluation of alexithymia and depression in severe obese patients not affected by eating disorders. Eating and Weight Disorders, 16, 24-29. doi: 10.1007/BF03327517.

Daniel, T. O., Stanton, C. M., \& Epstein, L. H. (2013). The future is now: comparing the effect of episodic future thinking on impulsivity in lean and obese individuals. Appetite, 71, 120125. doi: 10.1016/j.appet.2013.07.010.

Davies, H., Fox, J., Naumann, U., Treasure, J., Schmidt, U., \& Tchanturia, K. (2012). Cognitive remediation and emotion skills training (CREST) for anorexia nervosa: An observational study using neuropsychological outcomes. European Eating Disorders Review, 20, 211-217. doi: 10.1002/erv.2170.

Dingemans, A. E., Danner, U. N., Donker, J. M., Aardoom, J., van Meer, F. T., Obias, K., .. Van Furth, E.F. (2014). The effectiveness of cognitive remediation therapy in patients with a severe or enduring eating disorder: a randomized controlled trial. Psychotherapy Psychosomatic, 83, 29-36. doi: 10.1159/000355240

Fagundo, A., de la Torre, R., Jiménez-Murcia, S., Agüera, Z., Granero, R., Tárrega, S.,... Fernández-Aranda, F. (2012). Executive functions profile in extreme eating/weight conditions: from anorexia nervosa to obesity. PLOS One, 7, e43382. doi: https://doi.org/10.1371/journal.pone.0043382

Galimberti, E., Fadda, E, Cavallini, M. C., Martoni, R., Erzegovesi, S., \& Bellodi, L. (2013). Executive functioning in anorexia nervosa patients and their unaffected relatives. Psychiatry Research, 208, 238-244. doi: 10.1016/j.psychres. 2012.10.001.

Gearhardt, A., Frilo, C., Dileone, R., Brownell, K., \& Potenza, M. (2011). Can food be addictive? Public health and policy implications. Addiction, 106, 1208-1212. doi: 10.1111/j.1360-0443.2010.03301-x.

Giel, K., Hartmann, A., Zeeck, A., Jux, A., Vuck, A., Gierthmuehlen, P.,... Joos, A. (2016). Decreased emotional perception in obesity. European Eating Disorders Review, 24, 341-346. doi: 10.1002/erv.2444.

Giombini, L., Moynihan, J., Turco, M., \& Nesbitt, S. (2016). Evaluation of individual cognitive remediation therapy (CRT) for the treatment of young people with anorexia nervosa. Eating and Weight Disorders, 1-7. doi: 10.1007/s40519-016-0322-4.

Gionta, D. (1995). Food addiction. American Health, 14, 52-56.

Grant., D., \& Berg, E. (1948). A behavioural analysis of degree of reinforcement and ease of shifting to new responses in a weigl-type card sorting problem. Journal of Experimental Psychology, 38, 404-411.
Harrison, A., Mountford, V., \& Tchanturia, K. (2014). Social anhedonia and work and social functioning in the acute and recovered phases of eating disorders. Psychiatry Research, 218 , 187-94. doi: 10.1016/j.psychres.2014.04.007.

Kittel, R., Brauhardt, A., \& Hilbert, A. (2014). Cognitive and Emotional Functioning in Binge-Eating Disorder: A Systematic Review. International Journal of Eating Disorders, 48, 535-554. doi: 10.1002/eat.22419.

Leehr, E., Krohmer, K., Schag, K., Dresler, T., Zipfel, S., \& Giel, K. (2015). Emotion regulation model in binge eating disorder and obesity. A systematic review. Neuroscience and Biobehavioral Reviews, 125-134. doi: 10.1016/j.neubiorev.2014.12.008.

Lock, J., Agras, W. S., Fitzpatrick, K. K., Bryson, S. W., Jo, B., \& Tchanturia, K. (2013). Is outpatient cognitive remediation therapy feasible to use in randomized clinical trials for anorexia nervosa? International Journal of Eating Disorders, 46, 567-575. doi: 10.1002/eat.22134

Matsumoto, J., Hirano, Y., Numata, N., Matzuzawa, D., Murano, S., \&okote, K.,... Nakazato, M. (2015). Comparison in decision-making between bulimia nervosa, anorexia nervosa, and healthy women: Influence of mood status and pathological eating concerns. Journal of Eating Disorders, 3, 14. doi: 10.1186/s40337-015-0050-6.

Mezzich, J., Ruipérez, M., Pérez, C., Yoon, G., Liu, J., \& Mahmud, S. (2000). The spanish version of the Quality of life index: presentation and validation. The journal of nervous mental disease, 188, 301-305.

Ministerio de Sanidad, Servicios Sociales e Igualdad (2015). Informe anual del Sistema Nacional de Salud, 2012. Edición revisada, junio 2015. Disponible en www.msssi.gob.es

Mobbs, O., Iglesias, K., Golay, A., \& Van der Linden, M. (2011). Cognitive deficits in obese persons with and without binge eating disorder. Investigation using a mental flexibility task. Appetite, 57, 263-271. doi: 10.1016/j.appet.2011.04.023

Money, C., Davies, H., \& Tchanturia, K. (2011). A case study introducing cognitive remediation \& emotion skills training (CREST) for anorexia nervosa in-patient care. Clinical Case Studies, 10, 110-121. doi: 10.1177/1534650110396545

Money, C., Genders, B., Treasure, J., Schmidt, U., \& Tchanturia, K. (2011). A brief emotion focused intervention for in-patients with anorexia nervosa: A qualitative study. Journal of Health Psychology, 16, 947-958. doi: 10.1177/1359105310396395

National institute for health and clinical excellence (NICE) (2004). Eating disorders: Core interventions in the treatment and management of anorexia nervosa, bulimia nervosa and related eating disorders. London: National Institute for Clinical Excellence.

Neumark-Sztainer, D., Wall, M., Eisenberg, M.E., Story, M., \& Hannan, P.J. (2006). Overweight status and weight control behaviors in adolescents: Longitudinal and secular trends from 1999-2004. Preventive Medicine, 43, 52-9. doi: 10.1016/j. ypmed.2006.03.014

Neumark-Sztainer, D., Wall, M., Haines, J., Story, M., Sherwood, N., \& Van den Berg, P. (2007). Shared risk and protective factors for overweight and disordered eating in adolescents. American Journal of Preventive Medicine, 33, 359-369. doi: 10.1016/j.amepre.2007.07.031

Organización Mundial de la Salud (2016). Obesidad y sobrepeso. Recuperado de http://www.who.int/mediacentre/factsheets/ fs $311 /$ es/ 
Perpiñá, C., Segura, M., \& Sánchez, S. (2017). Cognitive flexibility and decision-making in eating disorders and obesity. Eating and Weight Disorders, 22, 435-444. doi: 10.1007/s40519-016-0331-3

Periáñez, J. A., y Barceló, F. (2001). Adaptación Madrid del test de clasificación de cartas de Wisconsin: un estudio comparativo de consistencia interna. Revista de Neurología, 337, 611-618.

Qavam, S., Anisan, A., Fathi, M., \& Pourab, A. (2015). Study of relationship between obesity and executive functions among high school students in Bushehr, Iran. Journal of Diabetes and Metabolic Disorders, 14, 79. doi: 10.1186/s40200-015-0211-9

Raman, J., Hay, P., \& Smith, E. (2014). Manualised cognitive remediation therapy for adult obesity: study protocol for a randomised controlled trial. Trials, 15, 426. doi: 10.1186/17456215-15-426.

Sandín, B., Chorot, P., Lostao, L., Joiner, T. E., Santed, M. A., y Valiente, R. M. (1999). Escalas PANAS de afecto positivo y negativo: validación factorial y convergencia transcultural. Psicothema, 11, 37-51.

Sandín, B., Chorot, P., Santed, M. A., y Jiménez, P. (1996). Dimensiones de la alexitimia y estados emocionales de ansiedad, depresión e ira. Psiquis, 17, 37-45.

Shaw, K., O'Rourke, P., Del Mar, C., \& Kenardy, J. (2005). Psychological interventions for overweight or obesity. The Cochrane Database Systematic Reviews, 18, CD003818. doi: 10.1002/14651858.CD003818.pub2

Stanton, A. L., García, M. E., \& Green, S. B. (1990). Development and validation of the Situational Appetite Measures. Addictive Behaviors, 15, 461-472.
Tchanturia, K., Davies, H., Roberts, M., Harrison, A., Nakazato, M., Schmidt, U.,... Morris, R. (2012). Poor cognitive flexibility in eating disorders: examining the evidence using the Wisconsin card sorting task. PLoS 7, e28331.

Tchanturia, K., \& Lock, J. (2011). Cognitive remediation therapy for eating disorders: Development, refinement and future directions. In R. Adan, \& W. Kaye (Eds). Behavioral Neurobiology of Eating Disorder, 6, (pp. 269-87). Heidelberg, Germany: Springer.

Tchanturia, K., Lloyd, S., \& Lang, K. (2013). Cognitive remediation in eating disorders. International Journal of Eating Disorders, 46, 492-496. doi: 10.1002/eat.22235.

Wadden, T.A., Butryn, M.L., \& Byrne, K.J. (2004). Efficacy of lifestyle modification for long-term weight control. Obesity Research, 12, 151-62. doi: 10.1038/oby.2004.282.

Watson, D., Clark, L., \& Tellegen, A. (1988). Development and validation of brief measures of positive and negative affect: the PANAS scales. Journal of Personality and Social Psychology, 54, 1063-1070.

Weider, S., Indredavik, M., lydersen, S., \& Hestad, K. (2016). Central coherence, visuoconstruction and visual memory in patients with eating disorders as measured by different scoring methods of the Rey complex figure test. European Eating Disorders Review, 24, 106-113. doi: 10.1002/erv.2385.

Witkin, H., Oltman, P., Raskin, E., \& Karp S. (1971). A manual for the Embedded figures test. Palo Alto CA: Consulting Psychologist. 